\title{
Tachyon cosmology, supernovae data, and the big brake singularity
}

\author{
Z. Keresztes \\ Department of Theoretical Physics, University of Szeged, Tisza Lajos krt 84-86, Szeged 6720, Hungary \\ Department of Experimental Physics, University of Szeged, Dóm Tér 9, Szeged 6720, Hungary \\ L. Á. Gergely \\ Department of Theoretical Physics, University of Szeged, Tisza Lajos krt 84-86, Szeged 6720, Hungary \\ Department of Experimental Physics, University of Szeged, Dóm Tér 9, Szeged 6720, Hungary \\ Department of Applied Science, London South Bank University, 103 Borough Road, London SE1 OAA, United Kingdom \\ V. Gorini and U. Moschella \\ Dipartimento di Scienze Fisiche e Mathematiche, Università dell'Insubria, Via Valleggio 11, 22100 Como, Italy \\ INFN, sezione di Milano, Via Celoria 16, 20133 Milano, Italy \\ A. Yu. Kamenshchik \\ Dipartimento di Fisica and INFN, via Irnerio 46, 40126 Bologna, Italy \\ L. D. Landau Institute for Theoretical Physics, Russian Academy of Sciences, Kosygin street 2, 119334 Moscow, Russia \\ (Received 15 January 2009; published 6 April 2009)
}

\begin{abstract}
We compare the existing observational data on type Ia supernovae with the evolutions of the Universe predicted by a one-parameter family of tachyon models which we have introduced recently [Phys. Rev. D 69, 123512 (2004)]. Among the set of the trajectories of the model which are compatible with the data there is a consistent subset for which the Universe ends up in a new type of soft cosmological singularity dubbed big brake. This opens up yet another scenario for the future history of the Universe besides the one predicted by the standard $\Lambda$ CDM model.
\end{abstract}

DOI: 10.1103/PhysRevD.79.083504

PACS numbers: 98.80.Cq, 98.80.Jk, 98.80.Es, 95.36.+x

\section{INTRODUCTION}

The discovery of cosmic acceleration [1] has stimulated the study of different models of dark energy [2] which may be responsible for such a phenomenon. Models of dark energy include those based on different perfect fluids, having negative pressure, on minimally and nonminimally coupled scalar fields and on fields having nonstandard kinetic terms [3,4]. The latter ones include as a subclass the models based on different forms of the Born-Infeldtype action, which is often associated with the tachyons arising in the context of string theory [5]. Tachyonic models with relatively simple potentials were confronted with observational data in [6]. Compared to the standard KleinGordon scalar field cosmological models the dynamics of tachyon models can be much richer due to the nonlinearity of the dependence of the tachyon Lagrangians on the kinetic term of the tachyon field.

In a recent paper [7] a particular one-parameter family of tachyon models was considered, which has revealed some unexpected features. At some values of the parameter of the model a long period of accelerated quasi-de Sitter expansion is followed by a period of cosmic deceleration culminating, after a finite time, in an encounter with a cosmological singularity of a new type, which was named big brake. This singularity is characterized by an infinite negative value of the second time derivative of the cosmological radius of the Universe, while its first time derivative and the Hubble variable vanish, and the radius itself acquires a finite value. This singularity belongs to the class of soft (sudden) cosmological singularities [8-10] which have been rather intensively studied during the last years. Here it is worth mentioning that in the context of the scrutiny of candidates for the role of dark energy, some other singularities attract the attention of cosmologists. Among them a special place is occupied by the big rip singularity [11], arising in some models where phantom dark energy [12] is present. The possibility of the existence of a phase of contraction of the Universe, ending up in the standard big crunch cosmological singularity, was also considered in the literature [13]. Recently, $w$ singularities were also proposed [14].

We may ask why the model proposed in [7] is worth studying. First, the soft (sudden) cosmological singularity of the big brake type arises in our model in a very natural way as a particular class of solutions of the dynamical system. Second, the model has another interesting feature. A subtle interplay between geometry and matter induces a change of the very nature of the latter: it transforms from a tachyon into a "pseudotachyon" field (see [7] for details). We point out that a similar effect was observed also in scalar-phantom cosmological models [15]. Phenomena of this kind represent a distinguishing feature of general relativity [16]: the requirement of self-consistency of Einstein equations can impose the form of the equations of motion for the matter. 
An attractive peculiarity of the tachyon model studied in Ref. [7] is the fact that there the big brake singularity is not put in "by hands" but arises naturally as a result of the cosmological evolution, provided some initial conditions are chosen. Therefore it is a consequence of the dynamics, rather than a pure kinematical possibility. Such evolution leading to the big brake coexists with another type of evolution describing an infinite expansion of the Universe. In other words, a small change of initial conditions can have drastic consequences for the future of the Universe. Actually, in spite of it being somewhat exotic, we show that the cosmological model [7] does not contradict observations. To this aim we compare the cosmological evolutions predicted in [7] with the data coming from the supernovae type Ia observations. We select the compatible initial conditions by studying the backward evolution in comparison with the luminosity—redshift diagrams for the supernovae type Ia standard(izable) candles. Then, choosing initial conditions which are compatible at the $1 \sigma$ level with the data, we study the forward evolution and show that a deceleration period following the present accelerated expansion is possible, and when it is so, we estimate how long it is expected to last.

The structure of the paper is the following. In Sec. II we introduce the model and its basic equations; in Sec. III we find a subset of initial conditions which are compatible with the observational data by integrating numerically the dynamical equations backwards in time; in Sec. IV we study numerically the cosmological evolutions for the selected initial conditions by numerical integration forward in time. We end with some concluding remarks.

\section{TACHYON COSMOLOGICAL MODEL}

We consider the flat Friedmann universe with the metric $d s^{2}=d t^{2}-a^{2}(t) d l^{2}$, filled with a spatially homogeneous tachyon field $T$ evolving according to the Lagrangian

$$
L=-V(T) \sqrt{1-g_{00} \dot{T}^{2}} .
$$

The energy density and the pressure of this field are, respectively,

$$
\varepsilon=\frac{V(T)}{\sqrt{1-\dot{T}^{2}}}
$$

and

$$
p=-V(T) \sqrt{1-\dot{T}^{2}} .
$$

The equation of motion for the tachyon is

$$
\frac{\ddot{T}}{1-\dot{T}^{2}}+3 \frac{\dot{a} \dot{T}}{a}+\frac{V_{, T}}{V}=0 .
$$

We consider the following tachyon potential $V(T)$ [7]:

$$
\begin{aligned}
V(T)= & \frac{\Lambda}{\sin ^{2}\left(\frac{3}{2} \sqrt{\Lambda(1+k)} T\right)} \\
& \times \sqrt{1-(1+k) \cos ^{2}\left(\frac{3}{2} \sqrt{\Lambda(1+k)} T\right)},
\end{aligned}
$$

where $\Lambda$ is a positive constant and $-1<k<1$.

Taking into account the Friedmann equation $H^{2}=\varepsilon$, where the Hubble variable $H$ is defined as $H \equiv \dot{a} / a$, and the Newtonian constant is normalized as $8 \pi G / 3=1$, we obtain the following dynamical system:

$$
\begin{gathered}
\dot{T}=s, \\
\dot{s}=-3 \sqrt{V}\left(1-s^{2}\right)^{3 / 4} s-\left(1-s^{2}\right) \frac{V_{, T}}{V} .
\end{gathered}
$$

When the parameter $k$ is negative, the evolution of the system (6) and (7) is confined inside the rectangle

$$
\begin{gathered}
-1 \leq s \leq 1, \\
0 \leq T \leq \frac{2 \pi}{3 \sqrt{\Lambda(1+k)}} .
\end{gathered}
$$

The system has only one critical point:

$$
T_{0}=\frac{\pi}{3 \sqrt{\Lambda(1+k)}}, \quad s_{0}=0,
$$

which is an attractive node corresponding to a de Sitter expansion with Hubble parameter

$$
H_{0}=\sqrt{\Lambda}
$$

All cosmological histories begin at the big bang type cosmological singularity located on the upper $(s=1)$ or lower $(s=-1)$ side of the rectangle (8) and (9), the individual history being parametrized by the initial value of $T$ satisfying the inequality (9). They all end up in the node (10).

In the case $k>0$ the situation is more complicated. First of all, the real potential $V$ is well-defined only in the interval

$$
T_{3} \leq T \leq T_{4},
$$

where

$$
T_{3}=\frac{2}{3 \sqrt{(1+k) \Lambda}} \arccos \frac{1}{\sqrt{1+k}}
$$

$$
T_{4}=\frac{2}{3 \sqrt{(1+k) \Lambda}}\left(\pi-\arccos \frac{1}{\sqrt{1+k}}\right) .
$$

The dynamical system (6) and (7) has three fixed points: the node (10) and the two saddle points with coordinates

$$
T_{1}=\frac{2}{3 \sqrt{(1+k) \Lambda}} \arccos \sqrt{\frac{1-k}{1+k}}, \quad s_{1}=0,
$$


and, respectively,

$$
T_{2}=\frac{2}{3 \sqrt{(1+k) \Lambda}}\left(\pi-\arccos \sqrt{\frac{1-k}{1+k}}\right), \quad s_{2}=0,
$$

which give rise to an unstable de Sitter regime with Hubble parameter $H_{1}=\sqrt{(1+k) \Lambda / 2 \sqrt{k}}>H_{0}$.

The most striking feature of the model under consideration with $k>0$ consists in the fact that now the cosmological trajectories do cross the corners of the rectangle (8) and (12). Indeed, the direct analysis of the system of differential equations in the vicinity of the points $P, Q$, $Q^{\prime}$ and $P^{\prime}$ (see Fig. 1) shows that these points are not singular points of the system [7]. Moreover, there is no cosmological singularity in these points [7]. That means that the cosmological evolutions must be continued through them. An apparent obstacle to such a continuation is the fact that the expression under the square root in the formula for the potential (5) changes sign when $T$ becomes smaller than $T_{3}$ or greater than $T_{4}$. However, the expression under the square root for the kinetic term $\sqrt{1-s^{2}}$ also changes sign at the same time. Then, since the Lagrangian of the theory is the product of these square roots, these simultaneous changes of sign leave the Lagrangian and the corresponding expressions for the energy density (2) and the pressure (3) real. The equation of motion for the tachyon field (4) also conserves its form. The sign, which we prescribe for the product (or for the ratio) of the square roots is uniquely determined by the Friedmann equation. In analyzing the behavior of our dynamical system in the regions where $|s|>1$ it is convenient to use the new potential

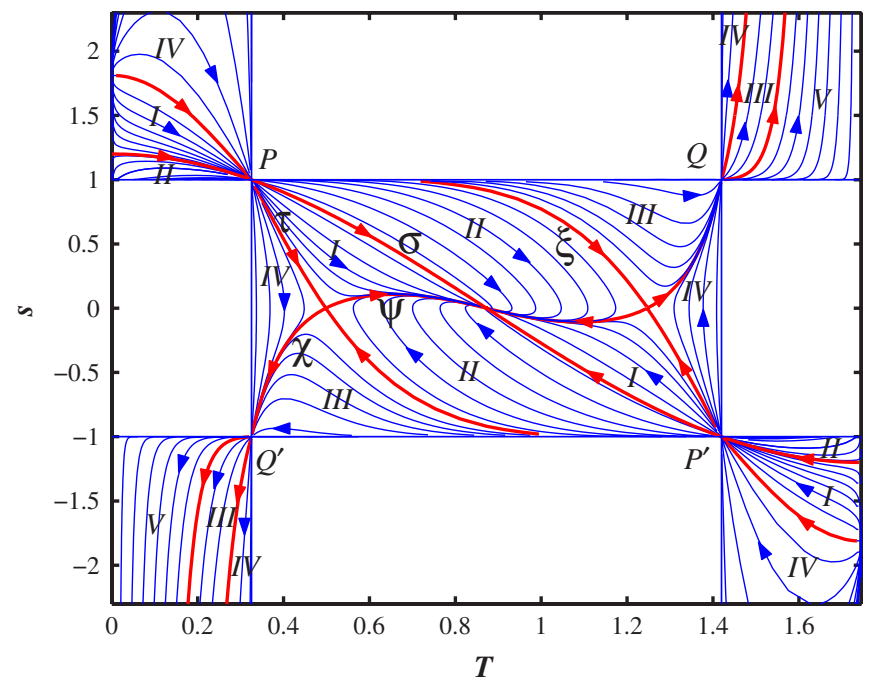

FIG. 1 (color online). Phase portrait evolution for $k>0(k=$ $0.44)$.

$$
\begin{aligned}
W(T)= & \frac{\Lambda}{\sin ^{2}\left(\frac{3}{2} \sqrt{\Lambda(1+k)} T\right)} \\
& \times \sqrt{(1+k) \cos ^{2}\left(\frac{3}{2} \sqrt{\Lambda(1+k)} T\right)-1},
\end{aligned}
$$

and to substitute in all expressions the term $1-s^{2}$ by $s^{2}-$ 1. In doing so the energy density and pressure have the form

$$
\varepsilon=\frac{W(T)}{\sqrt{s^{2}-1}}
$$

and

$$
p=W(T) \sqrt{s^{2}-1},
$$

respectively, being both positive.

The procedure of continuation of the trajectories through the corners of the rectangle is described in detail in [7]. Here, for the convenience of the reader we reproduce the phase portrait of the dynamical system from [7] with some brief comments. The rectangle in the phase space $(T, s)$ should be complemented by four infinite stripes (see Fig. 1). The left upper stripe (the right lower stripe) corresponds to the initial stages of the cosmological evolution, while the right upper stripe (the left lower stripe) corresponds to the final stages. There are five classes of qualitatively different cosmological trajectories. The trajectories belonging to classes I and II end their evolution with an infinite de Sitter expansion, while the trajectories of classes III, IV and V encounter a big brake singularity. The curves $\sigma, \xi, \tau, \psi$ and $\chi$ are separatrices, dividing different classes of trajectories.

We end this section with the following remark. Like the other tachyon or Dirac-Born-Infeld cosmological models (for example, models displaying the power-law or exponential potentials) the model based on potential (5) possesses a wide class of cosmological evolutions ending up in an infinite accelerated expansion. In addition, for small values of $T$, this potential behaves as $1 / T^{2}$, a behavior which has been widely studied in the literature. So far, so good. On the other hand, because of the more complicated structure of the potential (5), our model exhibits another class of trajectories with a qualitatively very different behavior and, in our opinion, this is precisely the feature which makes it particularly interesting.

\section{THE TACHYON COSMOLOGICAL MODEL AND COMPARISON WITH SUPERNOVAE TYPE IA OBSERVATIONAL DATA}

In this section we select, at the confidence level of $1 \sigma$ and for a given choice of values of the parameter $k$, the set of initial conditions $(z=0)$ for the system (6) and (7), which are compatible with the supernovae type Ia data taken from Ref. [17]. To this purpose, for the numerical 
analysis of the model it is convenient to rescale the relevant variables introducing the following dimensionless quantities:

$$
\hat{H}=\frac{H}{H_{0}}, \quad \hat{V}=\frac{V}{H_{0}^{2}}, \quad \Omega_{\Lambda}=\frac{\Lambda}{H_{0}^{2}}, \quad \hat{T}=H_{0} T,
$$

where $H_{0}$ is the present value of the Hubble parameter $H_{0}=H(z=0)$. In addition we find it convenient to replace the variable $T$ with the new variable

$$
y=\cos \left(\frac{3}{2} \sqrt{\Omega_{\Lambda}(1+k)} \hat{T}\right)
$$

and also to switch from the time derivative to the derivative with respect to the redshift $z$ :

$$
\frac{d}{d t}=-H(1+z) \frac{d}{d z},
$$

and denote $d / d z$ with a prime.

Then, the system of equations (6) and (7) in terms of the new variables $\hat{H}, s$, and $y$ (all depending on $z$ ) becomes

$$
\begin{gathered}
\hat{H}^{2}=\frac{\hat{V}}{\left(1-s^{2}\right)^{1 / 2}}, \\
s=\frac{2 y^{\prime}(1+z) \hat{H}}{3 \sqrt{\Omega_{\Lambda}(1+k)\left(1-y^{2}\right)}}, \\
(1+z) \hat{H} s^{\prime}=3 \sqrt{\hat{V}}\left(1-s^{2}\right)^{3 / 4} s+\left(1-s^{2}\right) \frac{\hat{V}_{, \hat{T}}}{\hat{V}},
\end{gathered}
$$

where $\hat{V}$ and $\hat{V}_{, T}$ are given by

$$
\begin{gathered}
\hat{V}=\frac{\Omega_{\Lambda}\left[1-(1+k) y^{2}\right]^{1 / 2}}{1-y^{2}}, \\
\hat{V}_{, \hat{T}}=\frac{3 \Omega_{\Lambda} \sqrt{\Omega_{\Lambda}(1+k)} y\left[k-1+(1+k) y^{2}\right]}{2\left(1-y^{2}\right)^{3 / 2}\left[1-(1+k) y^{2}\right]^{1 / 2}} .
\end{gathered}
$$

Since $\hat{H}^{2}(0)=1$, the present day values of the variables $s$ and $y$ satisfy the constraint

$$
s(0)= \pm \sqrt{1-\frac{\Omega_{\Lambda}^{2}\left[1-(1+k) y(0)^{2}\right]}{\left[1-y^{2}(0)\right]^{2}}} .
$$

We can avoid double coverage of the parameter space (the model being invariant under the simultaneous change of signs $y_{0} \rightarrow-y_{0}$ and $s_{0} \rightarrow-s_{0}$ ) by replacing $s_{0}$ by the new variable

$$
w_{0}=\frac{1}{1+s_{0}^{2}} .
$$

The luminosity distance function for a flat Friedmann universe

$$
d_{L}(z)=(1+z) \int_{0}^{z} \frac{d z^{*}}{H\left(z^{*}\right)}
$$

gives for the dimensionless luminosity distance $\hat{d}_{L}=$ $H_{0} d_{L}$ the equation

$$
\left(\frac{\hat{d}_{L}}{1+z}\right)^{\prime}=\frac{1}{\hat{H}}
$$

We are now in a position to compare our model with the supernovae type Ia data [17].

Following Ref. [18] we introduce the distance modulus type quantity $5 \log _{10} \hat{d}_{L}(z)+M$, with $M$ a constant offset between the data and the theoretical expression. The comparison involves computing

$$
\chi^{2}=\sum_{i=1}^{N} \frac{1}{\sigma_{i}^{2}}\left[5 \log _{10} \hat{d}_{L}^{\exp }\left(z_{i}\right)-M-5 \log _{10} \hat{d}_{L}\left(z_{i}\right)\right]^{2},
$$

where the sum is over the supernovae in the data set and $\sigma_{i}$ are the experimental errors in $5 \log _{10} \hat{d}_{L}^{\exp }\left(z_{i}\right)$. The distance luminosity function $\hat{d}_{L}(z)$ depends on the initial condition $y_{0}=y(0)$ and $s_{0}=s(0)$. We minimize this expression with respect to $M$ obtaining

$$
M=\frac{L}{D},
$$

with

$$
\begin{gathered}
L=\sum_{i=1}^{N} \frac{1}{\sigma_{i}^{2}}\left[5 \log _{10} \hat{d}_{L}^{\exp }\left(z_{i}\right)-5 \log _{10} \hat{d}_{L}\left(z_{i}\right)\right], \\
D=\sum_{i=1}^{N} \frac{1}{\sigma_{i}^{2}} .
\end{gathered}
$$

In Table I are listed the values $y_{j}, j=1,2,3,4$, of the variable $y$ corresponding to the values $T_{j}$ of the variable $T$ given in formulas (13)-(16) for the chosen positive values of $k$.

Since the expansion of the present day Universe is accelerated the pressure is negative, and hence $\left|s_{0}\right|<1$. Therefore, the initial point in the phase diagram $(T, s)$ should lie inside the rectangle $\left(T_{3}<T<T_{4},|s|<1\right)$ (see Fig. 1). Thus the bounds on the model are not satisfied in the ranges $y_{0}<y_{4}$ and $y_{0}>y_{3}$.

In Fig. 2 we represent the values of $\chi^{2}$ in the parameter plane of the initial conditions $\left[y_{0}=y(0), w_{0}=w(0)\right]$, for the choices $k=0, \pm 0.2, \pm 0.4$ and 0.6. The contours

TABLE I. The values of $y_{j}$ (corresponding to the $T_{j}$ ) for some positive values of $k$.

\begin{tabular}{lccc}
\hline \hline$k$ & 0.2 & 0.4 & 0.6 \\
\hline$y_{1,2}$ & \pm 0.816 & \pm 0.655 & \pm 0.500 \\
$y_{3,4}$ & \pm 0.913 & \pm 0.845 & \pm 0.791 \\
\hline \hline
\end{tabular}



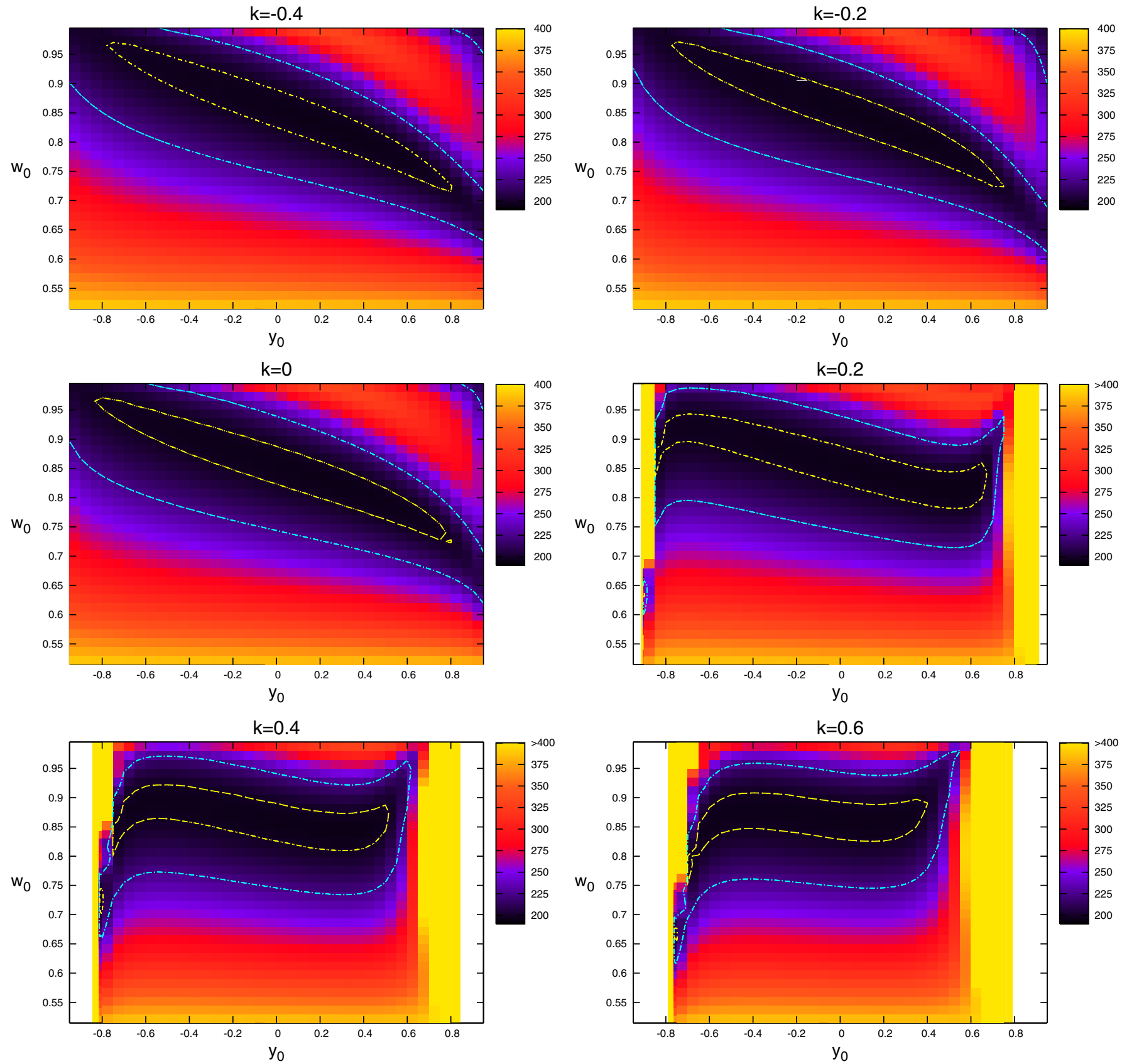

FIG. 2 (color online). The fit of the luminosity distance vs redshift for $k=-0.4$ (upper left), -0.2 (upper right), 0 (middle left), 0.2 (middle right), 0.4 (lower left), and 0.6 (lower right), in the parameter plane $\left[y_{0}, w_{0}=1 /\left(1+s_{0}^{2}\right)\right]$. The white areas represent regions where the bounds on the model are not satisfied. The contours refer to the $68.3 \%(1 \sigma)$ and $95.4 \%(2 \sigma)$ confidence levels. For increasing values of $|k|<1$ the well-fitting regions are increasingly smaller. The color code for $\chi^{2}$ is indicated on the vertical stripes.

represent the $68.3(1 \sigma)$ and $95.4(2 \sigma)$ confidence levels and the white areas are unallowed regions.

\section{FUTURE COSMOLOGICAL EVOLUTIONS}

In this section, in order to investigate the possible futures of the Universe within the tachyon cosmological model, we evolve numerically the model forward in time starting from the parameter range $\left(w_{0}, y_{0}\right)$ of initial conditions for which the fitting with the supernovae data is within $1 \sigma(68.3 \%)$ confidence level. We do this by numerical integration of equations of motion from $z=0$ towards negative values of $z$.

The results of these computations, corresponding to the six values of $k$ chosen earlier, are displayed in Fig. 3 in the space $\left(w=\left(1+s^{2}\right)^{-1}, y, z\right)$. The evolution curves start from the allowed region $\left(w_{0}, y_{0}\right)$ in the plane $z=0$. The final de Sitter state is characterized by the point $\left(w_{d S}=1\right.$, 

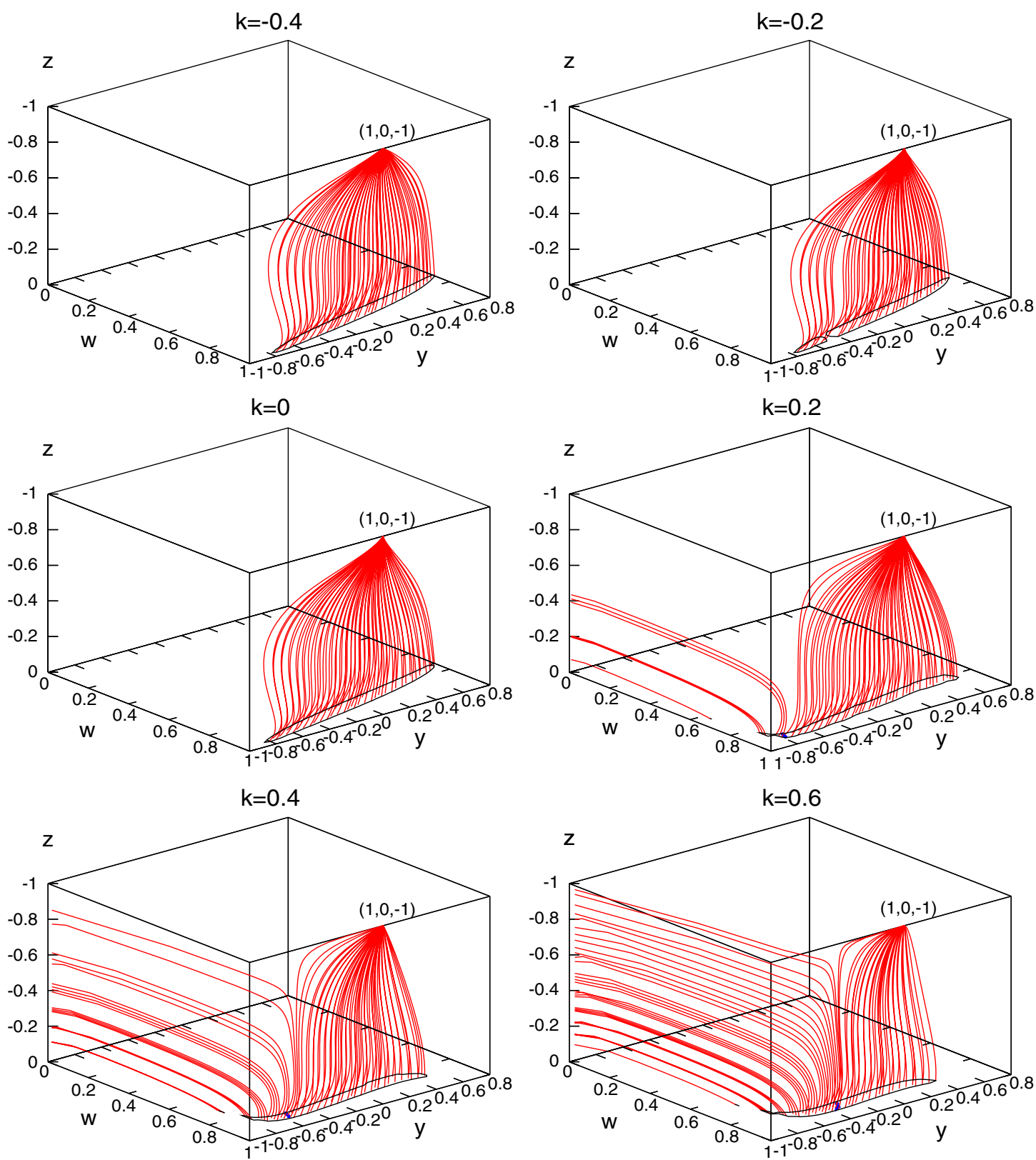

FIG. 3 (color online). The future evolution of those universes which are in a $68.3 \%$ confidence level fit with the supernova data. The $1 \sigma$ contours (black lines in the $z=0$ plane) are from Fig. 2 [the parameter plane $\left(y_{0}, w_{0}\right)$ is the $z=0$ plane here]. The sequence of figures and the values of $k$ are the same as in Fig. 2. The short and thick (blue) line in the plane of initial conditions separates the $1 \sigma$ parameter ranges for which the universe evolves into a de Sitter regime or towards the big brake singularity. Future evolutions towards the big brake singularity of the universes selected by the comparison with supernovae data become more frequent with increasing $k$.

$\left.y_{d S}=0, z_{d S}=-1\right)$, and the big brake final state by points $\left(w_{\mathrm{BB}}=0,-1<y_{\mathrm{BB}}<0,-1<z_{\mathrm{BB}}<0\right)$.

Whereas all trajectories with $k \leq 0$ end up eventually into the de Sitter state, those with $k>0$ can either evolve into the de Sitter state or into the big brake state, depending on the particular initial condition $\left(w_{0}, y_{0}\right)$. The fraction of curves eventually meeting a big brake increases with increasing $k$. This is clearly seen in Fig. 3 from the relative sizes of the $1 \sigma$ subdomains belonging to these two regimes, which are separated by a line.
For all future evolutions encountering a big brake singularity we have computed the actual time $t_{\mathrm{BB}}$ it will take to reach the singularity, measured from the present moment $z=0$, using the equation $\left(H_{0} t\right)^{\prime}=-\hat{H}^{-1}(1+z)^{-1}$. The results are shown in Tables II, III, and IV. In the tables the parameter values at which the pressure turns from negative to positive are also displayed.

Finally we have evolved numerically backward in time some of the trajectories crossing the $1 \sigma$ domain, until they reached one of the big bang singularities of the model. All 
TABLE II. Properties of the tachyonic universes with $k=0.2$ which (a) are within $1 \sigma$ confidence level fit with the type Ia supernova data and (b) evolve into a big brake singularity. Columns 1 and 2 represent a grid of values of the allowed model parameters. Columns 3 and 4: The redshift $z_{*}$ and time $t_{*}$ at the future tachyonic crossing (when $s=1$ and the pressure becomes positive). Columns 5 and 6: The redshift $z_{\mathrm{BB}}$ and time $t_{\mathrm{BB}}$ necessary to reach the big brake. The former indicates the relative size of the Universe when it encounters the big brake. (The values of $t_{*}$ and $t_{\mathrm{BB}}$ were computed with the Hubble parameter $H_{0}=73 \mathrm{~km} / \mathrm{s} / \mathrm{Mpc}$.)

\begin{tabular}{lccccc}
\hline \hline$y_{0}$ & $w_{0}$ & $z_{*}$ & $t_{*}\left(10^{9} \mathrm{yrs}\right)$ & $z_{\mathrm{BB}}$ & $t_{\mathrm{BB}}\left(10^{9} \mathrm{yrs}\right)$ \\
\hline-0.90 & 0.635 & -0.024 & 0.3 & -0.068 & 1.0 \\
-0.85 & 0.845 & -0.158 & 2.4 & -0.194 & 3.1 \\
-0.85 & 0.860 & -0.162 & 2.4 & -0.198 & 3.1 \\
-0.85 & 0.875 & -0.166 & 2.5 & -0.201 & 3.2 \\
-0.80 & 0.890 & -0.363 & 6.2 & -0.390 & 6.9 \\
-0.80 & 0.905 & -0.384 & 6.7 & -0.409 & 7.3 \\
-0.80 & 0.920 & -0.408 & 7.2 & -0.432 & 7.9 \\
\hline \hline
\end{tabular}

TABLE III. As in Table II, for $k=0.4$.

\begin{tabular}{lccccc}
\hline \hline$y_{0}$ & $w_{0}$ & $z_{*}$ & $t_{*}\left(10^{9} \mathrm{yrs}\right)$ & $z_{\mathrm{BB}}$ & $t_{\mathrm{BB}}\left(10^{9} \mathrm{yrs}\right)$ \\
\hline-0.80 & 0.710 & -0.059 & 0.8 & -0.106 & 1.6 \\
-0.80 & 0.725 & -0.059 & 0.8 & -0.105 & 1.6 \\
-0.80 & 0.740 & -0.060 & 0.8 & -0.105 & 1.6 \\
-0.75 & 0.815 & -0.144 & 2.1 & -0.184 & 2.9 \\
-0.75 & 0.830 & -0.147 & 2.2 & -0.187 & 3.0 \\
-0.75 & 0.845 & -0.150 & 2.2 & -0.189 & 3.0 \\
-0.70 & 0.845 & -0.241 & 3.8 & -0.276 & 4.6 \\
-0.70 & 0.860 & -0.248 & 4.0 & -0.282 & 4.7 \\
-0.70 & 0.875 & -0.256 & 4.1 & -0.290 & 4.9 \\
-0.70 & 0.890 & -0.264 & 4.2 & -0.298 & 5.0 \\
-0.65 & 0.860 & -0.358 & 6.2 & -0.387 & 7.0 \\
-0.65 & 0.875 & -0.372 & 6.5 & -0.400 & 7.2 \\
-0.65 & 0.890 & -0.388 & 6.8 & -0.415 & 7.6 \\
-0.65 & 0.905 & -0.406 & 7.2 & -0.432 & 8.0 \\
-0.60 & 0.875 & -0.521 & 10 & -0.542 & 11 \\
-0.60 & 0.890 & -0.551 & 11 & -0.571 & 12 \\
-0.60 & 0.905 & -0.587 & 12 & -0.605 & 13 \\
-0.55 & 0.875 & -0.756 & 19 & -0.766 & 20 \\
-0.55 & 0.890 & -0.837 & 25 & -0.845 & 26 \\
\hline \hline
\end{tabular}

trajectories we have checked originate from the singularity at $|s|=1$. In other words, they start from the horizontal boundaries of the rectangle in the phase plane $(T, s)$, and depending on whether they evolve into an infinite de Sitter expansion or reach the big brake singularity, they belong to either type II or III.

\section{CONCLUDING REMARKS}

In this paper we have shown that the tachyon cosmological model of Ref. [7] allows for a consistent set of
TABLE IV. As in Table II, for $k=0.6$. The evolutions into a big brake singularity compatible with supernova observations are more numerous with increasing $k$.

\begin{tabular}{llcccc}
\hline \hline$y_{0}$ & $w_{0}$ & $z_{*}$ & $t_{*}\left(10^{9} \mathrm{yrs}\right)$ & $z_{\mathrm{BB}}$ & $t_{\mathrm{BB}}\left(10^{9} \mathrm{yrs}\right)$ \\
\hline-0.75 & 0.665 & -0.039 & 0.5 & -0.088 & 1.4 \\
-0.70 & 0.755 & -0.098 & 1.4 & -0.145 & 2.3 \\
-0.70 & 0.770 & -0.100 & 1.5 & -0.145 & 2.3 \\
-0.70 & 0.785 & -0.101 & 1.5 & -0.146 & 2.3 \\
-0.70 & 0.800 & -0.102 & 1.5 & -0.146 & 2.3 \\
-0.65 & 0.815 & -0.168 & 2.6 & -0.209 & 3.4 \\
-0.65 & 0.830 & -0.171 & 2.6 & -0.212 & 3.4 \\
-0.65 & 0.845 & -0.175 & 2.7 & -0.215 & 3.5 \\
-0.60 & 0.830 & -0.240 & 3.9 & -0.277 & 4.7 \\
-0.60 & 0.845 & -0.247 & 4.0 & -0.283 & 4.8 \\
-0.60 & 0.860 & -0.254 & 4.1 & -0.289 & 4.9 \\
-0.60 & 0.875 & -0.261 & 4.2 & -0.296 & 4.0 \\
-0.55 & 0.845 & -0.325 & 5.5 & -0.357 & 6.3 \\
-0.55 & 0.860 & -0.335 & 5.7 & -0.366 & 6.5 \\
-0.55 & 0.875 & -0.347 & 5.9 & -0.377 & 6.7 \\
-0.55 & 0.890 & -0.359 & 6.2 & -0.389 & 7.0 \\
-0.50 & 0.845 & -0.411 & 7.5 & -0.439 & 8.3 \\
-0.50 & 0.860 & -0.427 & 7.8 & -0.453 & 8.6 \\
-0.50 & 0.875 & -0.444 & 8.2 & -0.469 & 9.0 \\
-0.50 & 0.890 & -0.463 & 8.6 & -0.488 & 9.4 \\
-0.45 & 0.860 & -0.533 & 10 & -0.554 & 11 \\
-0.45 & 0.875 & -0.557 & 11 & -0.577 & 12 \\
-0.45 & 0.890 & -0.584 & 12 & -0.603 & 13 \\
-0.45 & 0.905 & -0.616 & 13 & -0.633 & 14 \\
-0.40 & 0.860 & -0.658 & 15 & -0.673 & 16 \\
-0.40 & 0.875 & -0.693 & 16 & -0.707 & 17 \\
-0.40 & 0.890 & -0.733 & 18 & -0.745 & 19 \\
-0.40 & 0.905 & -0.779 & 21 & -0.789 & 22 \\
-0.35 & 0.860 & -0.814 & 23 & -0.822 & 24 \\
-0.35 & 0.875 & -0.865 & 28 & -0.872 & 29 \\
-0.35 & 0.890 & -0.927 & 36 & -0.930 & 37 \\
-0.30 & 0.845 & -0.955 & 43 & -0.957 & 44 \\
\hline \hline
\end{tabular}

trajectories which are compatible with the supernovae type Ia data.

We have found that, among these, for positive values of the parameter $k$ of the model, there is a subset of evolutions which end up into a big brake singularity and, for the latter, we have computed the relevant big brake parameters $z_{\mathrm{BB}}$ and $t_{\mathrm{BB}}$.

The compatibility of cosmological evolutions possessing soft cosmological singularities with the supernovae type Ia data was studied in [9]. Curiously, it was found in Ref. [9] that a sudden singularity may take place in already a very close future, even less than $10 \times 10^{6}$ years. However this analysis was purely kinematical, and we also note that the parameters in our model (as given by the tachyonic dynamics) near the big brake singularity fall outside the range considered in [9]. The problem of stability of a cosmological evolution in the vicinity of such singularities was studied in [10]. 
Thus, in spite of being a toy model, the tachyon cosmological model [7] can serve as a prototype of realistic (i.e. compatible with observational data) cosmological models which may lead to a final fate of the Universe, different from the infinite quasi-de Sitter expansion of the $\Lambda \mathrm{CDM}$ model. What will actually happen in the future is left to our far away descendants to experience.

\section{ACKNOWLEDGMENTS}

We thank Gy. Szabó for discussions in the early stages of this project. We are grateful to J.D. Barrow and M.P.
Dạbrowski for useful correspondence. Z. K. was supported by OTKA Grant No. 69036; L. Á. G. was supported by OTKA Grant No. 69036, the London South Bank University Research Opportunities Fund and the Polányi Program of the Hungarian National Office for Research and Technology (NKTH); A. K. was partially supported by RFBR Grant No. 08-02-00923 and by Grant No. LSS4899.2008.2.
[1] A. Riess et al., Astron. J. 116, 1009 (1998); S. J. Perlmutter et al., Astrophys. J. 517, 565 (1999).

[2] V. Sahni and A. A. Starobinsky, Int. J. Mod. Phys. D 9, 373 (2000); 15, 2105 (2006); T. Padmanabhan, Phys. Rep. 380, 235 (2003); P. J. E. Peebles and B. Ratra, Rev. Mod. Phys. 75, 559 (2003); E. J. Copeland, M. Sami, and S. Tsujikawa, Int. J. Mod. Phys. D 15, 1753 (2006).

[3] C. Armendariz-Picon, V.F. Mukhanov, and P.J. Steinhardt, Phys. Rev. D 63, 103510 (2001).

[4] A. Sen, J. High Energy Phys. 07 (2002) 065; G. W. Gibbons, Phys. Lett. B 537, 1 (2002); A. Feinstein, Phys. Rev. D 66, 063511 (2002); T. Padmanabhan, Phys. Rev. D 66, 021301 (2002); A. Frolov, L. Kofman, and A. Starobinsky, Phys. Lett. B 545, 8 (2002); N. Barnaby, J. High Energy Phys. 07 (2004) 025; R. Herrera, D. Pavon, and W. Zimdahl, Gen. Relativ. Gravit. 36, 2161 (2004); J. M. Aguirregabiria and R. Lazkoz, Mod. Phys. Lett. A 19, 927 (2004); A. Sen, Phys. Scr. T117, 70 (2005); E. J. Copeland, M. R. Garousi, M. Sami, and S. Tsujikawa, Phys. Rev. D 71, 043003 (2005); R. Lazkoz, Int. J. Mod. Phys. D 14, 635 (2005); V. Gorini, A. Kamenshchik, U. Moschella, V. Pasquier, and A. Starobinsky, Phys. Rev. D 72, 103518 (2005); E. Elizalde and J.Q. Hurtado, Int. J. Mod. Phys. D 14, 1439 (2005); C. Quercellini, M. Bruni, and A. Balbi, Classical Quantum Gravity 24, 5413 (2007); J. Martin and M. Yamaguchi, Phys. Rev. D 77, 123508 (2008).

[5] A. Sen, J. High Energy Phys. 04 (2002) 048.

[6] J. S. Bagla, H. K. Jassal, and T. Padmanabhan, Phys. Rev. D 67, 063504 (2003); G. Calcagni and A. R. Liddle, Phys. Rev. D 74, 043528 (2006); R. J. Yang, S. N. Zhang, and Y. Liu, J. Cosmol. Astropart. Phys. 01 (2008) 017.

[7] V. Gorini, A. Yu. Kamenshchik, U. Moschella, and V.
Pasquier, Phys. Rev. D 69, 123512 (2004).

[8] Y. Shtanov and V. Sahni, Classical Quantum Gravity 19, L101 (2002); J. D. Barrow, Classical Quantum Gravity 21, L79 (2004); 21, 5619 (2004); A. Kamenshchik, C. Kiefer, and B. Sandhofer, Phys. Rev. D 76, 064032 (2007).

[9] M. P. Dąbrowski, T. Denkiewicz, and M. A. Hendry, Phys. Rev. D 75, 123524 (2007).

[10] J. D. Barrow and S.Z.W. Lip, arXiv:0901.1626.

[11] A. A. Starobinsky, Gravitation Cosmol. 6, 157 (2000); R. R. Caldwell, M. Kamionkowski, and N. N. Weinberg, Phys. Rev. Lett. 91, 071301 (2003).

[12] R. R. Caldwell, Phys. Lett. B 545, 23 (2002).

[13] R. Kallosh, A. Linde, S. Prokushkin, and M. Shmakova, Phys. Rev. D 66, 123503 (2002); R. Kallosh and A. Linde, J. Cosmol. Astropart. Phys. 02 (2003) 002; U. Alam, V. Sahni, and A. A. Starobinsky, J. Cosmol. Astropart. Phys. 04 (2003) 002; R. Kallosh, J. Kratochvil, A. Linde, E. V. Linder, and M. Shmakova, J. Cosmol. Astropart. Phys. 10 (2003) 015.

[14] M.P. Dąbrowski and T. Denkiewicz, arXiv:0902.3107 [Phys. Rev. D (to be published)].

[15] A. A. Andrianov, F. Cannata, and A. Y. Kamenshchik, Phys. Rev. D 72, 043531 (2005); F. Cannata and A. Y. Kamenshchik, Int. J. Mod. Phys. D 16, 1683 (2007).

[16] A. Einstein, L. Infeld, and B. Hoffmann, Ann. Math. 39, 65 (1938); A. Einstein and L. Infeld, Ann. Math. 41, 455 (1940); C. Lanczos, Phys. Rev. 59, 813 (1941); V. Fock, JETP 9, 375 (1939).

[17] W. M. Wood-Vasey et al. (ESSENCE Collaboration), Astrophys. J. 666, 694 (2007).

[18] D. A. Dicus and W. W. Repko, Phys. Rev. D 70, 083527 (2004). 